\title{
Genetik und Epigenetik in der Psychotherapie von Depression und Angststörungen
}

\author{
Svenja Schaumburg ${ }^{a}$ Silvia Schneider ${ }^{b}$ Jürgen Margraf ${ }^{a}$ Robert Kumsta ${ }^{c}$ \\ André Wannemüller ${ }^{a}$ \\ ${ }^{a}$ Klinische Psychologie und Psychotherapie, Fakultät für Psychologie, Ruhr-Universität Bochum, Bochum, Germany; \\ ${ }^{b}$ Klinische Kinder- und Jugendpsychologie, Fakultät für Psychologie, Ruhr-Universität Bochum, Bochum, Germany; \\ 'Genetic Psychology, Fakultät für Psychologie, Ruhr-Universität Bochum, Bochum, Germany
}

Schlüsselwörter

Biomarker · Genetik · Epigenetik · Therapiegenetik

\section{Zusammenfassung}

Obwohl heute für die meisten psychischen Störungen über die gesamte Lebensspanne hinweg wirksame psychotherapeutische Behandlungsangebote zur Verfügung stehen, besteht immer noch eine zu große Variabilität in Bezug auf den kurz- und langfristigen Erfolg solcher Interventionen. Das Feld der Therapiegenetik beschäftigt sich mit der Vorhersage des Erfolges psychotherapeutischer Behandlungen durch genetische oder epigenetische Marker. Dabei wird das Ziel verfolgt, zukünftig psychotherapeutische Interventionen auf der Basis solcher Marker individualisiert anzuwenden und dadurch die Effektivität der Behandlungsangebote weiter zu verbessern. In diesem Beitrag wird der aktuelle Stand der Forschung in diesem Feld dargestellt, methodologische Herausforderungen skizziert und die Entwicklung der noch jungen Disziplin, ausgehend von theoriegeleiteten Kandidatengenstudien, hin zu hypothesenfreien genomweiten Assoziationsstudien und der Verwendung polygener Risiko-Scores, nachgezeichnet. Der zweite Abschnitt dieses Übersichtsartikels widmet sich epigenetischen Prozessen und diskutiert kritisch erste Befunde, die sich mit Modifikation von DNA-Methylierungsmustern im Zuge psychotherapeutischer Intervention beschäftigen.

(c) 2020 S. Karger AG, Basel

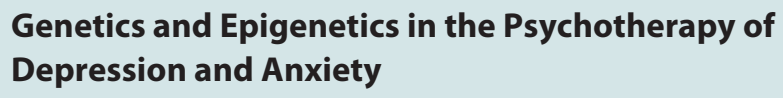

\section{Keywords}

Biomarker · Genetics · Epigenetics · Therapygenetics

\section{Abstract}

Although effective psychotherapeutic treatment is now available for most mental disorders throughout the life span, there is still large variability in the short and longterm success of such interventions. The field of therapygenetics is concerned with predicting the success of psychotherapeutic treatments using genetic or epigenetic markers. The long-term goal is to individualize future psychotherapeutic interventions on the basis of such markers to further improve the effectiveness of treatment options. In this review, we present the current state of research, outline methodological challenges, and trace the development of this still young discipline from theorybased candidate gene studies to hypothesis-free genome-wide association studies. In the second part of this

Dr. André Wannemüller

Lehrstuhl für Klinische Psychologie und Psychotherapie

Forschungs- und Behandlungszentrum für Psychische Gesundheit

Ruhr-Universität Bochum, Massenbergstrasse 9-13, DE-44787 Bochum (Germany)

E-Mail andre.wannemueller@ rub.de

Prof. Dr. Robert Kumsta

Fakultät für Psychologie, Genetic Psychology, IB E5/117 - Postfach 20

Ruhr-Universität Bochum

Universitätsstrasse 150, DE-44801 Bochum (Germany)

E-Mail robert.kumsta@ rub.de 
review, epigenetic processes are discussed, with a focus on recent studies investigating intervention-associated modifications of DNA methylation patterns.

(c) 2020 S. Karger AG, Basel

\section{Einleitung}

Für die Behandlung der meisten psychischen Störungen stehen heute für die gesamte Lebensspanne anhaltend wirksame kognitiv-verhaltenstherapeutische Behandlungsansätze zur Verfügung. Bei Angst- und affektiven Störungen, also zwei der häufigsten Klassen psychischer Störungen bei Erwachsenen, zeigen z.B. ca. $50 \%$ der Patienten eine vollständige Remission nach der Behandlung [Loerinc et al., 2015]. Des Weiteren erleben viele Patienten eine teilweise Verbesserung ihrer Symptome, selbst dann, wenn diese zuvor schon seit Jahrzehnten bestanden [DeRubeis et al., 2005; Loerinc et al., 2015]. Im Umkehrschluss bedeuten diese Zahlen aber auch, dass nicht jedem Patienten durch eine Psychotherapie geholfen wird und es eine große Variabilität bezüglich des Therapieerfolgs gibt. Befunde zur Wirksamkeit der Psychotherapie bei Kindern, gemittelt über alle Störungen, Alters- und Verfahrensklassen, ergeben Posttreatment und Follow-up (FU) Effektstärken von Cohen's $d=0,46$, respektive 0,38 , auf mittlerem Niveau und unterstreichen die Befunde auch für diesen Altersbereich [Weisz et al., 2017]. In randomisiert kontrollierten Studien bei Erwachsenen zeigen sich Raten des Ansprechens auf eine kognitiv-verhaltenstherapeutische Behandlung von $38-82 \%$ [Hofmann et al., 2012].

In Anbetracht dieser Variabilität im Behandlungserfolg sollte es ein vorrangiges Ziel der psychotherapeutischen Forschung und Praxis sein, Wirksamkeitsnachweise für alle eingesetzten Verfahren zu liefern und im Falle fehlender, nicht ausreichender oder sogar negativer Evidenz konsequent auf deren Anwendung zu verzichten. Außerdem gilt es, Therapiemethoden zu entwickeln, die individuelle Patientenmerkmale besser berücksichtigen und deshalb für den jeweiligen Patienten optimal geeignet sind. Diese Individualisierung würde viel Zeit und Ressourcen für jede Behandlung sparen und die Wahrscheinlichkeit erhöhen, dass ein Patient von der gewählten Intervention profitiert. Beispielsweise könnte so auch die Frage beantwortet werden, für welche Individuen bereits eine Intervention auf niedrigem Niveau, das heißt mit z.B. wenigen Stunden oder geringerem Therapeutenkontakt, ausreicht und für welche eine Kombination aus verschiedenen Behandlungsoptionen notwendig erscheint [Eley, 2014]. Der Ansatz der "stratifizierten Medizin" [Trusheim et al., 2007] verfolgt diesen Grundgedanken und versucht, Biomarker zu identifizieren, die den Behandlungserfolg vorhersagen, und diese zu nut-
Kasten 1. Biomarker

Ein Biomarker wird allgemein als quantifizierbares Merkmal definiert, das als Indikator biologischer Prozesse, pathologischer Prozesse und pharmakologischer Reaktionen auf Interventionen beurteilt wird. Dieses Merkmal wird objektiv gemessen und kann zur Vorhersage der Inzidenz oder des Ausgangs einer Erkrankung sowie der Effekte einer Behandlung oder einer unbeabsichtigten Umweltexposition genutzt werden. Biomarker dienen als Surrogate klinisch bedeutsamer Endpunkte und korrelieren nicht notwendigerweise mit dem Erleben des Individuums [Strimbu und Tavel, 2010].

zen, um die Therapie individuell auf den Patienten abzustimmen und eine optimale Behandlung zu gewährleisten (Box 1). Im Gegensatz zur personalisierten Medizin erfolgt die Anpassung auf Basis bekannter Charakteristika spezifischer Patientenpopulationen und nicht des einzelnen Patienten. Durch solche Biomarker können dann klinische Prädiktoren des Therapieerfolgs (z.B. das Vorliegen einer komorbiden Störung) sinnvoll ergänzt werden [Hudson et al., 2013]. In der Psychotherapieforschung sind bislang einige wenige solcher Biomarker insbesondere für den Bereich der Angstbehandlungen identifiziert worden, beispielsweise die Funktion des anterioren cingulären Kortex oder die kardiovaskuläre Flexibilität bzw. Herzratenvariabilität [Lueken et al., 2016]. Das Ziel des vorliegenden Artikels ist, einen Überblick über bestehende Studien zu geben, die genetische und epigenetische Marker für Prädiktion und Validierung des Erfolgs von psychotherapeutischen Interventionen nutzen.

\section{Genetik in der Psychotherapie}

Der Begriff "therapygenetics" wurde erstmals 2012 von Eley et al. [2012] geprägt und bezieht sich auf die Vorhersage des Erfolgs psychotherapeutischer Behandlungen durch genetische Marker. Das konzeptuelle Rahmenmodell für diesen Ansatz wurde aus der Forschung zur GenUmwelt-Interaktion heraus entwickelt. Bereits in den 1970er Jahren postulierte Rutter [1972], dass die Sensitivität gegenüber Umweltreizen durch genetische Faktoren mitbestimmt wird. Lange Zeit herrschte das DiatheseStress-Modell vor, welches besagt, dass eine spezifische genetische Ausstattung in Kombination mit der Exposition gegenüber ungünstigen Umwelteinflüssen das Risiko für die Entwicklung von psychischen Störungen erhöht [Monroe und Simons, 1991]. Eine Erweiterung des Diathese-Stress-Modells wurde durch das Modell der differentiellen Suszeptibilität erreicht [Belsky und Pluess, 2009]. Demzufolge haben bestimmte Individuen nicht nur ein genetisch erhöhtes Risiko, eher maladaptive Reaktionen auf ungünstige Umweltbedingungen zu zeigen, sondern gleichzeitig eine höhere Wahrscheinlichkeit, auf 
bereichernde Umweltbedingungen mit positiven Entwicklungsergebnissen zu reagieren. Dieses Prinzip des "for better and for worse" ist von einem evolutionären Standpunkt aus gesehen sinnvoll. In Anbetracht sich verändernder und unvorhersehbarer Umwelten, kann eine genetische Disposition zu erhöhter oder erniedrigter Umweltsensitivität die Überlebensfähigkeit eines Individuums verbessern [Belsky and Pluess, 2009].

Das Feld der Psychotherapiegenetik betrachtet im Rahmen einer solchen Gen-Umwelt-Interaktion den Kontext der psychotherapeutischen Intervention als positive Umweltbedingung und untersucht die Rolle von Genen für die Prädiktion des Ergebnisses solcher Behandlungen [Lester and Eley, 2013]. Da die Umweltvariable im psychotherapeutischen Kontext bewusst manipuliert wird, handelt es sich somit im experimentellen Sinne um eine kontrollierte Bedingung. Dies erleichtert die Interpretation der Befunde im Vergleich zu retrospektiv erfassten Umwelterfahrungen (wie z.B. kindlicher Misshandlung), da eine prospektive Erfassung der Symptome vor der Umwelterfahrung, d.h. der Behandlung, möglich ist.

$\mathrm{Zu}$ Anfang fokussierten Therapiegenetikstudien zunächst auf die Variation einzelner Gene, sogenannter Kandidatengene, um den Erfolg einer Intervention vorherzusagen. Eine der ersten Studien lieferte mit einer Varianzaufklärung von $4 \%$ vielversprechende Ergebnisse [Bakermans-Kranenburg et al., 2008]. Es stellte sich heraus, dass vor allem Kinder mit dem 7-repeat-Allel des Dopamin-D4-Rezeptorgens (DRD4) von einer Intervention zur Förderung positiven Elternverhaltens profitierten und eine Reduktion externalisierender Auffälligkeiten zeigten, wohingegen Kinder ohne dieses Allel weniger auf die Intervention ansprachen.

Anhand dieser Studie lässt sich verdeutlichen, welche Kriterien für die Auswahl bestimmter Kandidatengene herangezogen werden. Im beschriebenen Fall des DRD4 hatte sich zuvor herausgestellt, dass das 7-repeat Allel mit ADHS in Verbindung gebracht werden kann [Faraone et al., 1999] und dass nur bei Kindern mit dem 7-repeat Allel, das mit einer geringeren Dopaminrezeptorsensitivität in Verbindung steht, Zusammenhänge zwischen elterlicher Insensitivität und externalisierenden Auffälligkeiten vorliegen. Biomarker bzw. Kandidatengene, die zur Vorhersage von Therapieerfolg herangezogen werden, leiten sich gemäß des Leitsatzes "cause informs cure" demnach aus Variablen ab, die an der Entstehung einer bestimmten Störung beteiligt sind. Dass es diesen genetischen Beitrag zur Störungsentwicklung von affektiven Störungen und Angststörungen gibt, zeigt sich an deren moderater Heritabilität [Lau and Eley, 2010]. Kandidatengenstudien wurden bis zum heutigen Tag mit einer Vielzahl von Genen durchgeführt, z.B. Brain-Derived Neurotrophic Factor (BDNF), Nervenwachstumsfaktor
(Nerve Growth Factor, NGF) oder Catechol-O-Methyltransferase (COMT). Es zeigte sich allerdings meist eine große Heterogenität in den Ergebnissen, und die Befunde konnten oftmals nicht repliziert werden [Eley, 2014]. Dies soll am Beispiel des häufigen Polymorphismus im Promoter des Serotonintransportergens (5HTTLinked Polymorphic Region; 5-HTTLPR) verdeutlicht werden, der als der meist untersuchte genetische Polymorphismus im Bereich der Angst- und depressiven Störungen gilt [Lester and Eley, 2013]. Er liegt in einer kurzen (S) und einer langen (L) Allelvariante vor. Im Vergleich zur L-Variante wurde für die S-Variante eine deutlich niedrigere Genexpression nachgewiesen [Lesch et al., 1996]. Für Träger der S-Variante konnte in verschiedenen Studien eine stärkere Reaktion auf negative Erfahrungen (z.B. chronischer Stress, traumatische Erlebnisse in der Kindheit) und infolge auch eine erhöhte Vulnerabilität für psychische Erkrankungen gezeigt werden [Caspi and Moffitt, 2006]. Metaanalysen zeigen hierzu sowohl kongruente [Karg et al., 2011] als auch nicht kongruente Befunde [Risch et al., 2009; Border et al., 2019]. Auf der anderen Seite waren Kinder mit homozygoter S-AllelAusstattung gegenüber der Entwicklung psychischer Erkrankungen vergleichsweise resistent, wenn sie in sehr günstigen und fördernden Umständen aufwuchsen [Taylor et al., 2006].

In Bezug auf den Erfolg psychotherapeutischer Behandlungen ergaben bislang drei Studien einen positiven Effekt des S-Allels auf den Behandlungserfolg: In einer Untersuchung von Eley et al. [2012] durchliefen Kinder mit verschiedenen Angststörungen eine kognitive Verhaltenstherapie (KVT), und es stellten sich Unterschiede zwischen SS- und SL-/LL-Trägern im Behandlungserfolg nach 6 Monaten heraus. $\mathrm{Zu}$ diesem Zeitpunkt waren 20\% mehr SS-Allel-Träger remittiert und zeigten einen Rückgang ihrer primären Angststörung. Übereinstimmend berichteten Kohen et al. [2011], dass SS-Allel-Träger, im Gegensatz zu LL-Allel-Trägern, von einer verhaltenstherapeutischen Behandlung bei einer Depression nach Schlaganfall profitierten. Außerdem ergab eine Untersuchung mit Patienten mit Panikstörung und Agoraphobie nach einer einwöchigen expositionsbasierten Behandlung, dass LL-Allel-Träger eine geringere Reduktion ihrer agoraphobischen Ängste erfuhren als hetero- oder homozygote S-Allel Träger [Knuts et al., 2014].

Im Gegensatz dazu stellte sich bei Patienten mit posttraumatischer Belastungsstörung (PTBS) nach 8 Sitzungen individueller KVT heraus, dass zur Nachfolgeuntersuchung nach 6 Monaten mehr Patienten mit S-Allel nach wie vor die Kriterien der PTBS erfüllten und dass diese im Durchschnitt mehr Traumasymptome aufwiesen.

Die meisten Studien zum 5-HTTLPR zeigten allerdings keine Prädiktion des Behandlungserfolgs durch
60

Verhaltenstherapie 2020;30:58-67 DOI: $10.1159 / 000505440$
Schaumburg/Schneider/Margraf/Kumsta/ Wannemüller 
Tabelle 1. Methodische Schwächen bisheriger Studien

- Heterogene Störungen (zwischen den einzelnen Studien, teilweise aber auch innerhalb der Studien, z.B. unterschiedliche Angststörungen)

- Unterschiedliche Definition von Therapieerfolg

O Remission der primären Störung

O Remission aller Störungen

O Rückgang der Symptome (Selbst- oder Fremdeinschätzung)

O Zeit bis zum Rückfall (Depression)

- Unterschiedliche Therapiemethoden

O Einzel-KVT

O Gruppen-KVT

O Elterngeleitete Intervention

O Internetbasierte Intervention

- Unterschiedliche Intensität und Dauer der Intervention

- Zusätzliche Medikation - medikationsfreie Patienten

- Heterogene Altersgruppen

verschiedene Allel-Varianten. Dies zeigte sich bei Patienten mit Panikstörung nach 10-wöchiger internet- oder gruppenbasierter KVT [Lonsdorf et al., 2010], bei sozial ängstlichen Probanden nach 15-wöchiger internet- oder gruppenbasierter KVT [Hedman et al., 2012] sowie bei Patienten mit rezidivierender Depression nach einer KVT [Bockting et al., 2013], um nur einige Beispiele zu nennen. Auch eine Replikation des Befundes bei Kindern mit Angststörung gelang nicht [Lester et al., 2016]. In dieser groß angelegten Studie wurden 829 Patienten mit Einzel-KVT, Gruppen-KVT oder Eltern-gestützter geleiteter Selbsthilfe behandelt, wobei die Stichprobengröße ausgereicht hätte, um sogar einen noch kleineren Effekt, als den in der Studie von Eley et al. [2012] berichteten, zu entdecken. Nach einer Zusammenführung der Patienten aus beiden Kohorten [Lester et al., 2016] zeigte sich nur ein signifikantes Ergebnis: SS-Allel-Träger wiesen zum FU eine höhere Remissionswahrscheinlichkeit bezüglich aller Angststörungen (jedoch nicht hinsichtlich der primären Angststörung) auf.

Die inkonsistenten Befunde der oben beschriebenen Studien können maßgeblich durch zu kleine Stichproben und eine methodische Heterogenität erklärt werden (Tabelle 1). Zum einen handelt es sich bei den zu erwartenden Effekten um sehr kleine, sodass die meisten beschriebenen Studien nicht genug Power haben, diese zu entdecken. Empfehlenswert wäre daher eine Zusammenarbeit verschiedener Forschergruppen, die ihre Patientengruppen unter der Voraussetzung aggregieren, dass die therapeutische Intervention unter hoch-standardisierten Bedingungen erfolgt, um einheitliche methodische Voraussetzungen zu schaffen. In einer kürzlich veröffentlichten Studie von Wannemüller et al. [2018] wurde genau diesem Aspekt besondere Beachtung geschenkt. 222 nicht-medizierte Patienten mit unterschiedlichen situativen Ängsten durchliefen eine standardisierte Ein-Sitzungs-Großgruppenbehandlung. Dabei zeigten sich keine Unterschiede im Therapieerfolg, wenn man den Zeitpunkt unmittelbar nach der Behandlung betrachtet. Im Zeitraum nach der Behandlung war allerdings bei LL-Allel-Trägern ein weiterer Rückgang ihrer Furcht zu beobachten, während sich das Furchtlevel von SL-Allel-Trägern im Vergleich zur Messung unmittelbar nach der Behandlung nicht veränderte. Im Gegensatz dazu zeigte sich bei SS-Allel-Trägern eine Rückkehr der Furcht.

Aufgrund bisheriger Befunde, die darauf hinweisen, dass genetische Einflüsse auf Verhaltensmerkmale individuell klein und über das Genom verteilt sind und somit einzelne Gene nur einen geringen Anteil an Varianz des Merkmals erklären [Chabris et al., 2015], entwickelt sich das Feld aktuell von einem Kandidatengenansatz hin $\mathrm{zu}$ einem genomweiten Ansatz weiter [Rayner et al., 2019]. Dazu haben zuletzt auch neue Möglichkeiten der Genomanalysetechnik beigetragen, durch die genomweite Auswertungen bei großen Stichproben möglich werden. Die erste genomweite Assoziationsstudie (GWAS) zum Therapieerfolg der KVT wurde von Coleman et al. [2016] bei 980 Kindern mit Angststörung durchgeführt, wobei auch in dieser Studie die Daten aus verschiedenen Behandlungszweigen (Einzeltherapie, Gruppentherapie, geleitete Selbsthilfe) aggregiert wurden. Es konnten hinsichtlich des Therapieerfolgs, gemessen an der Veränderung der klinischen Beurteilung des Schweregrades, sowohl zum Post-treatment- als auch zum FU-Zeitpunkt, keine Einzelbasenaustausche (Single Nucleotide Polymorphisms; SNPs) auf genomweitem Signifikanzlevel (alle $p<5 \times$ $10^{-8}$ ) identifiziert werden, die mit dem Behandlungserfolg assoziiert waren.

In der bisher größten GWAS zum Ergebnis psychotherapeutischer Interventionen [Rayner et al., 2019] wurden drei Kohorten zusammengefasst, die jeweils eine kognitiv-verhaltenstherapeutisch orientierte Behandlung erhielten: erwachsene Patienten mit Angststörung, erwachsene Patienten mit Major Depression und Kinder und Jugendliche mit Angststörung. Auch in dieser Studie konnten keine SNPs identifiziert werden, die mit dem Therapieergebnis in Verbindung stehen, wobei einige Loci unter dem liberaler angesetzten Signifikanzniveau für eine suggestive Assoziation lagen $\left(p<10^{-5}\right)$. Anzumerken ist hierbei, dass die Gesamtstichprobe aufgrund ihrer Größe von $N$ = 2'724 über $80 \%$ Power verfügte, Varianten zu finden, die 1,5\% der Varianz erklären. Zusammenfassend lässt sich also sagen, dass die Stichprobengrößen bestehender GWAS heute noch zu klein sind, um die mit Therapieerfolg assoziierten SNPs und die assoziierten individuell kleinen bis sehr kleinen Effekte zu identifizieren.

Eine Möglichkeit, die Ergebnisse großer GWAS zu nutzen, liegt in der Berechnung polygener Risiko-Scores 
Kasten 2. Genomweite Assoziationsstudien und polygene Scores

Bei genomweiten Assoziationsstudien (GWAS) handelt es sich um einen explorativen Ansatz, der darauf abzielt, Assoziationen zwischen genetischen Varianten und einem interessierenden Merkmal zu identifizieren. Dabei wird das gesamte Genom hypothesenfrei untersucht, um auch Varianten einschließen zu können, die nicht an der vermeintlichen Ätiologie der Störung beteiligt sind und somit noch nicht Gegenstand von Kandidatengenstudien wurden. Für jeden Genort wird untersucht, ob es einen Zusammenhang zwischen Allelhäufigkeit und der Ausprägung des Merkmals gibt. Da hierbei für multiples Testen korrigiert werden muss, erfordern GWAS immense Stichprobengrößen [Visscher et al., 2017]. Eine Möglichkeit, die Ergebnisse aus GWAS zu nutzen, bietet die Berechnung polygener Scores (PGS). Aus GWAS ist bekannt, wie stark der Zusammenhang zwischen Single Nucleotide Polymorphisms (SNPs) und dem untersuchten Merkmal ist. In einer unabhängigen Stichprobe wird für jede Person die Anzahl der Risikoallele in einem SNP bestimmt und mit der in GWAS ermittelten Effektstärke der Variante multipliziert. Anschließend werden die Werte über alle relevanten SNPs summiert (alternativ werden nur SNPs mit einbezogen, deren Assoziation mit dem Merkmal ein bestimmtes Signifikanzniveau unterschreitet, oder man bestimmt empirisch den optimalen Schwellenwert) und es ergibt sich für jede Person ein Summenscore, der sogenannte PGS [Martin et al., 2019]. Auf diese Weise können GWAS-Statistiken auch in kleineren Stichproben genutzt werden.

(Box 2). Hierbei werden Merkmale betrachtet, die aufgrund theoretischer Überlegungen eine Beziehung zum Therapieerfolg aufweisen könnten [z.B. Persönlichkeitsmerkmale, Bildungserfolg, Psychopathologie; Rayner et al., 2019].

In der Studie von Rayner et al. [2019] konnte der Therapieerfolg in einer meta-analytischen Zusammenfassung der erwähnten drei Stichproben durch keinen polygenen Score (PGS) für die oben genannte Merkmale vorhergesagt werden, was auf eine geringe genetische Kovarianz zwischen Merkmalen für Psychopathologie, Persönlichkeit und Bildungserfolg mit dem Therapieergebnis schließen lässt. Allerdings war ein hoher PGS für subjektives Wohlbefinden in der Stichprobe der Kinder und Jugendlichen mit Angststörung mit einem besseren Therapieergebnis assoziiert, und es lag ein Zusammenhang zwischen einem höheren genetischen Risiko für $\mathrm{Au}$ tismus und einem schlechteren Therapieergebnis in der Depressions-Stichprobe vor [siehe auch Anderson et al., 2019]. In der zuvor erwähnten Studie bei Kindern mit Angststörung [Coleman et al., 2016] konnte kein Einfluss der PGS für Major Depression, Schizophrenie und das Ansprechen auf Antidepressiva auf den Behandlungserfolg festgestellt werden.

Beispielhaft dafür, wie aus dem Zusammenspiel aus GWAS und PGS ein Erkenntnisgewinn resultieren kann, ist die Studie von Keers et al. [2016], in der zunächst genetische Varianten erkundet wurden, die mit einer er- höhten Umweltsensitivität einhergehen. In einer unabhängigen Stichprobe wurde dann ein PGS der Umweltsensitivität berechnet und überprüft, ob dieser PGS vorhersagen konnte, von welcher Intervention (wenig intensiv vs. intensiv) Kinder und Jugendliche mit Angststörung am meisten profitierten. Es stellte sich dabei heraus, dass Kinder mit hohem PGS für Umweltsensitivität eine intensivere Behandlung benötigen, um ein positives Therapieergebnis zu zeigen.

Abschließend lässt sich aus den bisher diskutierten Studien zu GWAS und PGS schließen, dass für zukünftige Studien größere Patientenpopulationen benötigt werden, um die zu erwartenden kleinen Effekte zu entdecken [siehe oben; Rayner et al., 2019]. Des Weiteren empfiehlt es sich, über verschiedene Studien hinweg den Therapieerfolg einheitlich $\mathrm{zu}$ definieren sowie Interventionen ähnlich zu gestalten, um eine Vergleichbarkeit der Befunde und eine mögliche metaanalytische Zusammenfassung zu ermöglichen [Lester und Eley, 2013].

Bei den gegebenen methodischen Empfehlungen sollte allerdings das Ziel der Ansätze, die prädiktive genetische oder andere biologische Marker untersuchen, nicht aus den Augen verloren werden. Wenn ein biologischer Prädiktor zur Entscheidungsfindung in Bezug auf die Therapieindikation beitragen soll, muss dieser gegenüber der Heterogenität von Störungsbildern robust sein, da diese das Bild in der klinischen Praxis widerspiegelt [Coleman et al., 2016]. Zukünftig könnten zunächst große multizentrische Studien, in denen streng manualisierte Behandlungen durchgeführt werden, um sowohl eine ausreichende Stichprobengröße als auch eine erhöhte Standardisierung der Behandlung zu gewährleisten, dazu dienen, Marker zu identifizieren. Im Anschluss kann dann überprüft werden, ob sich diese Effekte auch unter weniger standardisierten Bedingungen und bei heterogeneren Patientengruppen als relevant herausstellen. Nichtsdestotrotz sollte grundsätzlich das Ziel verfolgt werden, auf eine homogene Definition des Phänotyps hinzuarbeiten, was unter anderem durch die Betrachtung einzelner Merkmale anstelle komplexer Störungsgruppen gewährleistet werden könnte.

\section{Epigenetik in der Psychotherapie}

Epigenetik ist ein Sammelbegriff für eine Vielzahl von Mechanismen, die an der Genregulation beteiligt sind (Box 3). Epigenetische Prozesse spielen eine wichtige Rolle bei der Embryonalentwicklung und der Reifung des Organismus [Stewart et al., 2016], und sie werden zunehmend als eine entscheidende Schnittstelle in der Interaktion zwischen Genetik und Umweltfaktoren wahrgenommen. Studien an Modellorganismen haben gezeigt, dass ein Teil der epigenetischen Mechanismen sich nicht nur
Schaumburg/Schneider/Margraf/Kumsta/ Wannemüller 
Kasten 3. Epigenetik

$\mathrm{Zu}$ den wichtigsten epigenetischen Mechanismen gehören die DNA-Methylierung und Histonmodifikationen, wobei eine Reihe von weiteren Mechanismen bekannt sind [Allis und Jenuwein, 2016]. Bei DNA-Methylierung handelt es sich um eine chemische Modifikation der DNA, ohne dass die zu Grunde liegende Sequenz der Basen verändert wird. Methyliert werden hauptsächlich Cytosine (C), auf die ein Guanin (G) folgt, genannt CpG-Stellen. Der Zusammenhang zwischen CpG-Methylierung und der Auswirkung auf die Genexpression ist komplex und unter anderem abhängig von der genomischen Lokalisation. Höhere DNA-Methylierung in regulatorischen Regionen kann das Ablesen von Genen verringern, wobei DNA-Methylierung in einer kodierenden Genregion die Genexpression verstärken kann. Bei der Histonmodifikation handelt es sich um Modifikationen der Proteine, um die die DNA-Doppelhelix herum gepackt liegt. Die Modifikationen resultieren entweder in einem weniger dichten "Verpackungsgrad" der DNA um die Proteine, die das Ablesen der Gene erleichtert, oder in einer stärkeren DNAProtein-Verbindung, die das Ablesen von Genen verhindert.

im Laufe eines Lebens, sondern auch durch spezifische Umwelteinflüsse wie Umweltgifte und Ernährung verändert [Jirtle und Skinner, 2007].

Diese Befunde und vor allem die Studien am Rattenmodell, die erstmalig zeigen konnten, dass psychosoziale Einflüsse epigenetische Prozesse dauerhaft beeinflussen können [Weaver et al., 2004], haben dazu geführt, dass die Epigenetik verstärkt an Relevanz für die Psychologie und Psychopathologie gewinnt.

Die Pionierarbeit zu mütterlicher epigenetischer Programmierung hat gefunden, dass Nachkommen von Ratten mit geringem Brutpflegeverhalten später unter Stressbedingungen eine stärkere Reaktivität und eine verzögerte Hemmung der hormonellen Stressachse (Hypothalamus-Hypophysen-Nebennieren-Achse) zeigen. Diese Veränderung der Stressregulation konnte kausal durch eine höhere DNA-Methylierung und Histonacetylierung in der Promotorregion des Glukokortikoidrezeptorgens im Hippocampus erklärt werden [Weaver et al., 2004]. Eine genetische Übertragung konnte über Kreuzaufzucht ausgeschlossen werden; die epigenetischen Veränderungen wurden über die Interaktion zwischen Muttertier und Nachkommen weitergegeben. Ähnliche Befunde konnten für weitere, an der Stressregulation beteiligte Gene gefunden werden [Murgatroyd et al., 2009; Chen et al., 2012].

Seither werden epigenetische Prozesse als Mechanismus, oder zumindest als Korrelate, der "biologischen Einbettung" psychosozialer Einflussfaktoren auch im Humanbereich diskutiert. Es ist erwiesen, dass sowohl pränatale Belastungen (mütterlicher Stress, psychische Erkrankungen, Rauchen und Mangelernährung) sowie aversive Erfahrungen in der frühen Kindheit, wie Miss- brauch und Vernachlässigung, mit einem erhöhten Risiko für spätere psychische Beeinträchtigungen einhergehen [Gluckman und Hanson, 2004; Gilbert et al., 2009; Schlotz und Phillips, 2009; Sonuga-Barke et al., 2017]. Stabile Veränderungen genregulatorischer Prozesse, die in frühen Entwicklungsphasen durch epigenetische Modifikationen erzeugt wurden, stellen ein plausibles Erklärungsmodell für die Beobachtung langfristiger negativer Folgen nach Exposition gegenüber ungünstigen psychosozialen Einflussfaktoren in vulnerablen Entwicklungsphasen dar.

Allerdings liegen bislang nur wenige Studien vor, die den Zusammenhang zwischen frühkindlichem Stress, epigenetischen Modifikationen und späterer psychischer Belastung beim Menschen untersucht haben. Ferner zeichnen die bislang vorliegenden Humanstudien ein, im Vergleich zu den Tiermodellen, deutlich komplexeres Bild der Wechselwirkung zwischen menschlichem Epigenom und Umwelteinflüssen. Ein grundlegendes Problem bei epigenetischen Studien am Menschen ist die Untersuchung von peripherem Zellmaterial, in der Regel bestehend aus Blutzellen, Speichel oder Wangenepithel. Epigenetische Prozesse sind jedoch höchst zellspezifisch, und der Nachweis für eine funktionelle Bedeutung solcher peripheren Veränderungen der DNA-Methylierung für Prozesse im Gehirn steht noch aus. Systematische Vergleiche zwischen der DNA-Methylierung in Blutzellen und jener in Zellen aus verschiedenen Gehirnarealen konnten zeigen, dass die DNA-Methylierung in Blutzellen kein guter Prädiktor für jene in Hirngeweben ist nichtsdestotrotz waren die interindividuellen Unterschiede über die Gewebetypen hinweg stabil, so dass periphere DNA-Methylierungsmuster durchaus als nützliche Biomarker angesehen werden können [Hannon et al., 2015]. Außerdem sprechen Studien an Mäusen und Rhesusaffen dafür, dass DNA-Methylierungsmuster zumindest teilweise zwischen Immunzellen und Hirnzellen vergleichbar sind [Provençal et al., 2012; Massart et al., 2016].

Eine weitere naturgemäße Einschränkung ist, dass die Exposition gegenüber aversiven Erfahrungen nicht experimentell manipulierbar ist. Studien, die sich "natürlicher Experimente" bedienen, wie beispielsweise mütterlicher Mangelernährung während der Schwangerschaft im holländischen Hungerwinter [Tobi et al., 2018], oder institutioneller Deprivation wie im Falle ehemaliger rumänischer Heimkinder [Kumsta et al., 2016], deuten auf eine Assoziation zwischen psychosozialen Belastungen während der Embryonalentwicklung beziehungsweise in der frühen Kindheit und langfristig veränderter DNA-Methylierung hin. Weitere Befunde zu Effekten von pränatalem Stress auf Methylierungsmuster liegen vor zu mütterlicher Angststörung oder Depression [Hompes et al., 2013] sowie durch Naturkatastrophen ausgelöstem [Cao- 
Lei et al., 2014] oder selbstberichtetem mütterlichem Stress [Rijlaarsdam et al., 2016]. Auch in Bezug auf die Exposition gegenüber postnatalen Belastungen, wie z.B. frühkindlichem Stress [Essex et al., 2013], Misshandlungen in der Familie [Cecil et al., 2016], Armut [Borghol et al., 2012] und Bullying [Ouellet-Morin et al., 2013], wurden Effekte nachgewiesen. Aufgrund der Heterogenität der Studiendesigns sowie der untersuchten Risikofaktoren ist die Belastbarkeit der Ergebnisse jedoch schwer einzuschätzen, zumal auch eine epigenomweite Assoziationsstudie vorliegt, die bei einer umfangreichen Stichprobe $(N>1$ '600) keine Assoziation zwischen negativen Kindheitserfahrungen in der frühen Kindheit oder Adoleszenz und DNA-Methylierung zeigt [Marzi et al., 2018]. Trotz der heterogenen Befundlage nehmen epigenetische Modifikationen wahrscheinlich auch beim Menschen eine funktional bedeutsame Rolle ein. Allerdings ist man derzeit noch weit davon entfernt, einen epigenetischen Fingerabdruck aversiver früher Erfahrungen oder späterer traumatischer Erlebnisse zu identifizieren.

\section{DNA-Methylierung als Marker in der Psychotherapie}

Es wurde lange gemeinhin angenommen, dass DNAMethylierungsmuster in frühen Entwicklungsphasen etabliert werden und danach relativ stabil bleiben. Eine Reihe von Studien konnte allerdings zeigen, dass die DNAMethylierung dynamischer zu sein scheint als zunächst angenommen und sich in Abhängigkeit von internen (physiologischen) und umweltbedingten Signalen ändern kann [Wong et al., 2010; Dekkers et al., 2016; Joehanes et al., 2016; Saunderson et al., 2016; Emeny et al., 2018]. Die Tatsache, dass DNA-Methylierungsmuster über die gesamte Lebensspanne hinweg durch Umwelteinflüsse modifizierbar scheinen, hat Studien inspiriert, die der Frage nachgehen, ob DNA-Methylierungsmuster durch psychologische Interventionen veränderbar sind. Neben möglichen therapieassoziierten Veränderungen ist auch die Frage nach dem prädiktiven Wert der DNAMethylierung für den Therapieverlauf relevant.

Die wenigen bislang durchgeführten Studien verfolgten Kandidatengenansätze [Übersicht siehe Kumsta, 2019] und untersuchten die Methylierung von Genen, die an der Stressregulation beteiligt sind [NR3C1, FKBP5; Yehuda et al., 2013, Roberts et al., 2015, 2019], sowie von häufig in Assoziationsstudien untersuchten Genen, die für den Serotonintransporter (SLC6A4 [Roberts et al., 2014]), die Monoaminoxidase A (MAOA [Ziegler et al., 2016]) und den $B D N F$ [Perroud et al., 2013] kodieren. So konnte z.B. in der Studie von Ziegler et al. [2016] gezeigt werden, dass eine vergleichsweise niedrige Methylierung des MAOA-Gens mit der von Panikstörungspatienten vor einer Behandlung berichteten Symptomschwere assoziiert war. Bei Patienten, die auf die Behandlung ansprachen, wuchs die DNA-Methylierung nach der Be- handlung bis auf das Niveau einer gesunden Kontrollgruppe an. Im Gegensatz dazu fiel bei Patienten, die durch die Therapie nicht profitieren konnten, das Niveau der MAOA-Methylierung weiter ab.

Dieser Befund bildet ein gemeinsames Merkmal aller bisherigen Untersuchungen $\mathrm{ab}$, das darin besteht, dass Patienten, die von der jeweiligen Therapie profitierten, im Vergleich zu solchen, die keine Verbesserung zeigten, gegenläufige Muster in der Veränderung der DNA-Methylierung aufwiesen. Die bisherigen Studien haben jedoch noch Pilotcharakter und unterscheiden sich in Bezug auf Störungsbilder (PTBS, Angststörungen, Borderline Persönlichkeitsstörung), Therapieform und die Altersspanne der Patienten, so dass zum jetzigen Zeitpunkt eine studienübergreifende Einschätzung des Wertes von DNA-Methylierungsmustern als Marker für den Erfolg psychotherapeutischer Behandlungen noch schwierig ist.

Um klarere Aussagen zu ermöglichen, müssen zukünftige Studien zusätzlich für natürliche Fluktuationen der DNA-Methylierung über die Zeit kontrollieren. Bei Studien, für die das Genmaterial aus einer Vielzahl unterschiedlicher Zelltypen gewonnen wird, ist zudem zu kontrollieren, ob die gemessenen Unterschiede in der DNA-Methylierung nicht lediglich Änderungen in der Zellzusammensetzung widerspiegeln. So ist z.B. die $\mathrm{Zu}-$ sammensetzung des zirkulierenden Leukozytenpools nachweislich dynamisch und variiert z.B. stressbedingt [Richlin et al., 2004; Cole, 2010]. Die beobachteten Veränderungen müssen daher nicht zwangsläufig Prozesse der zellulären Umprogrammierung repräsentieren. Zur Vorsicht ruft hierbei auch die Tatsache, dass die beobachtete Methylierungsveränderung oft sehr klein und die biologische Signifikanz solch kleiner Veränderungen im Rahmen der Zellprogrammierung bislang noch unklar ist.

\section{Zusammenfassung und Fazit}

Die Forschung zu genetischen und v.a. epigenetischen Markern in der Psychotherapie steckt noch in den Kinderschuhen. Kandidatengenstudien haben bislang zumeist den Einfluss von Genen auf den Behandlungserfolg untersucht, die für die Ätiologie der behandelten Erkrankung relevant sind oder mit differentieller Suszeptibilität für Umwelteinflüsse in Zusammenhang gebracht werden. Einige Studien zeigten Effekte einzelner Genvarianten (z.B. in Bezug auf den 5-HTTLPR), die meisten Studien lieferten jedoch Nullbefunde, und die Ergebnisse waren oft inkonsistent und schlecht replizierbar. Angesichts der komplexen, polygenen Natur von Störungen wie Angst oder Depression, bei denen einzelne genetische Varianten nur extrem kleine Anteile der Varianz erklä-
64

Verhaltenstherapie 2020;30:58-67 DOI: $10.1159 / 000505440$
Schaumburg/Schneider/Margraf/Kumsta/ Wannemüller 
ren, ist dies jedoch nicht verwunderlich, und die meisten der existierenden Studien dürften daher zu klein gewesen sein, um einzelne, mit Therapieerfolg assoziierte genetische Varianten zu identifizieren. GWAS erlauben eine hypothesenfreie Testung, welche Genvarianten mit einem bestimmten Merkmal wie dem Therapieerfolg in Verbindung stehen. Bislang konnte jedoch noch kein genomweit signifikanter Marker in Bezug auf Erfolg psychotherapeutischer Interventionen identifiziert werden. Die Qualität von Kandidatengenstudien und GWAS leidet zurzeit noch darunter, dass häufig auf unterschiedliche Weise behandelte Patienten mit sehr heterogenen Störungsbildern zusammengefasst wurden, um größere Stichproben zu erhalten.

Im Falle des Vorliegens ausreichend großer GWAS für mit dem Therapieerfolg assoziierte Eigenschaften bringt die Verwendung von PGS den Vorteil mit sich, dass auch in verhältnismäßig kleinen Stichproben der Einfluss der genetischen Disposition für solche Traits auf den Therapieerfolg untersucht werden kann. Ergebnisse erster Untersuchungen unter Verwendung von PGS weisen in die Richtung, dass auch Veranlagungen für Eigenschaften, die in keinem augenscheinlichen Zusammenhang mit der behandelten Störung stehen (wie z.B. das Bildungsniveau), mit dem Behandlungserfolg in Verbindung stehen können und daher der Leitsatz "cause informs cure" wahrscheinlich zu kurz greift.

Die Modifizierbarkeit von DNA-Methylierungsmustern durch Umwelteinflüsse und der vermutete mediierende Einfluss der Effekte negativer Erfahrungen auf verschiedene Indikatoren psychischer Krankheit und Gesundheit rücken auch epigenetische Prozesse ins Interesse von Therapiegenetikstudien. Erste Studien zu epigenetischen Markern des Erfolgs psychotherapeutischer Behandlungen sind vielversprechend, sehen sich aber unterschiedlichen methodologischen Herausforderungen gegenüber, wie z.B. der starken Zellspezifität von Methylierungsmustern und der, je nach untersuchtem Zellgewebe, stark heterogenen und zuweilen stressbedingt fluktuierenden Zellzusammensetzung.

Bislang ist der Erkenntnisgewinn aus Therapie(epi)genetikstudien für die Indikationsentscheidung und Wahl des optimalen Therapieverfahrens im Sinne einer "personalisierten Psychotherapie" folglich noch als gering zu beurteilen. Aufgrund der genannten unterschiedlich gelagerten methodologischen Schwierigkeiten konnte das Potential dieses Feldes jedoch bislang auch bei Weitem noch nicht vollständig ausgeschöpft werden. Die Sammlung, Auswertung und der Zugang zu genomweiten Daten wird zukünftig immer einfacher werden, z.B. durch Populationsstudien, die neben gesundheitsrelevanten Daten auch genetische Daten erheben (z.B. UK Biobank). Die große Herausforderung von Therapiegenetikstudien besteht jedoch eher auf Seiten des zu untersuchenden
Phänotyps. Hier sollte zukünftig der Erfolg psychotherapeutischer Interventionen einheitlicher und genauer erfasst werden, die Behandlung stärker kontrolliert und bei hinsichtlich ihrer Symptomatik homogenen Patientengruppen appliziert werden. Wenn diese Kriterien in multizentrischen Studien umgesetzt werden, können GWAS von immer größeren (und gleichzeitig homogenen) Stichproben profitieren.

Zusammenfassend lässt sich festhalten, dass die genetischen Einflüsse auf das Ansprechen auf psychotherapeutische Intervention - wie bei anderen komplexen Phänotypen auch - extrem polygener Natur sind. Da die Effekte einzelner Varianten verschwindend gering sind, ist ein möglicher prädiktiver Nutzen genetischer Marker nur über PGS denkbar, die über den Summenwert dieser Effektstärken die genetische Disposition für das Ansprechen auf Psychotherapie widerspiegeln. Der prädiktive Wert von PGS wiederum ist abhängig von der Qualität und Stichprobengröße der zu Grunde liegenden GWAS. Wenn nun die oben beschriebenen Hindernisse überwunden werden, sind ausreichend große und für Therapieerfolg spezifische GWAS denkbar, die zur Identifikation valider genetischer Marker und daraus abgeleiteten PGS führen können. Es wird sich zeigen, inwieweit diese dann genügend Varianz an der beobachtbaren Variabilität im Behandlungserfolg aufklären, um auf der Basis genetischer Marker optimal wirksame personalisierte Therapieansätze zu entwickeln.

\section{Statement of Ethics}

Im Zusammenhang mit dieser Arbeit bestehen keine ethischen Konflikte.

\section{Disclosure Statement}

Die Autoren bestätigen, dass keiner der Autoren einen Interessenkonflikt hat.

Literatur Allis CD, Jenuwein T. The molecular hallmarks of epigenetic control. Nat Rev Genet. 2016 Aug; 17(8):487-500.

Anderson JS, Shade J, DiBlasi E, Shabalin AA, Docherty AR. Polygenic risk scoring and prediction of mental health outcomes. Curr Opin Psychol. 2019 Jun;27:77-81.

Bakermans-Kranenburg MJ, Van IJzendoorn MH, Pijlman FT, Mesman J, Juffer F. Experimental evidence for differential susceptibility: dopamine D4 receptor polymorphism (DRD4 VNTR) moderates intervention effects on toddlers' externalizing behavior in a randomized controlled trial. Dev Psychol. 2008 Jan; 44(1):293-300.

Belsky J, Pluess M. Beyond diathesis stress: differential susceptibility to environmental influences. Psychol Bull. 2009 Nov;135(6):885-908. 
Bockting CL, Mocking RJ, Lok A, Koeter MW, Schene AH. Therapygenetics: the 5HTTLPR as a biomarker for response to psychological therapy? Mol Psychiatry. 2013 Jul;18(7):744-5.

Border R, Johnson EC, Evans LM, Smolen A, Berley N, Sullivan PF, et al. No Support for Historical Candidate Gene or Candidate Geneby-Interaction Hypotheses for Major Depression Across Multiple Large Samples. Am J Psychiatry. 2019 May;176(5):376-87.

Borghol N, Suderman M, McArdle W, Racine A, Hallett M, Pembrey M, et al. Associations with early-life socio-economic position in adult DNA methylation. Int J Epidemiol. 2012 Feb;41(1):62-74.

Cao-Lei L, Massart R, Suderman MJ, Machnes Z, Elgbeili G, Laplante DP, et al. DNA methylation signatures triggered by prenatal maternal stress exposure to a natural disaster: Project Ice Storm. PLoS One. 2014 Sep;9(9):e107653.

Caspi A, Moffitt TE. Gene-environment interactions in psychiatry: joining forces with neuroscience. Nat Rev Neurosci. 2006 Jul;7(7):583-90.

Cecil CA, Smith RG, Walton E, Mill J, McCrory EJ, Viding E. Epigenetic signatures of childhood abuse and neglect: implications for psychiatric vulnerability. J Psychiatr Res. 2016 Dec;83:184-94.

Chabris CF, Lee JJ, Cesarini D, Benjamin DJ, Laibson DI. The Fourth Law of Behavior Genetics. Curr Dir Psychol Sci. 2015 Jul;24(4): 304-12.

Chen J, Evans AN, Liu Y, Honda M, Saavedra JM, Aguilera G. Maternal deprivation in rats is associated with corticotrophin-releasing hormone $(\mathrm{CRH})$ promoter hypomethylation and enhances CRH transcriptional responses to stress in adulthood. J Neuroendocrinol. 2012 Jul;24(7):1055-64.

Cole SW. Elevating the perspective on human stress genomics. Psychoneuroendocrinology. 2010 Aug;35(7):955-962.

Coleman JR, Lester KJ, Keers R, Roberts S, Curtis $\mathrm{C}$, Arendt $\mathrm{K}$, et al. Genome-wide association study of response to cognitive-behavioural therapy in children with anxiety disorders. $\mathrm{Br}$ J Psychiatry. 2016 Sep;209(3):236-43.

Dekkers KF, van Iterson M, Slieker RC, Moed $\mathrm{MH}$, Bonder $\mathrm{MJ}$, van Galen $\mathrm{M}$, et al.; BIOS Consortium. Blood lipids influence DNA methylation in circulating cells. Genome Biol. 2016 Jun;17(1):138

DeRubeis RJ, Hollon SD, Amsterdam JD, Shelton RC, Young PR, Salomon RM, et al. Cognitive therapy vs medications in the treatment of moderate to severe depression. Arch Gen Psychiatry. 2005 Apr;62(4):409-16.

Eley TC, Hudson JL, Creswell C, Tropeano M, Lester KJ, Cooper P, et al. Therapygenetics: the 5HTTLPR and response to psychological therapy. Mol Psychiatry. 2012 Mar;17(3):236-7.

Eley TC. The future of therapygenetics: where will studies predicting psychological treatment response from genomic markers lead? Depress Anxiety. 2014 Aug;31(8):617-20.

Emeny RT, Baumert J, Zannas AS, Kunze S, Wahl $S$, Iurato S, et al. Anxiety Associated Increased CpG Methylation in the Promoter of Asb1: A Translational Approach Evidenced by Epidemiological and Clinical Studies and a Murine Model. Neuropsychopharmacology. 2018 Jan;43(2):342-53.
Essex MJ, Boyce WT, Hertzman C, Lam LL, Arm strong JM, Neumann SM, et al. Epigenetic vestiges of early developmental adversity: childhood stress exposure and DNA methylation in adolescence. Child Dev. 2013 Jan-Feb; 84(1):58-75.

Faraone SV, Biederman J, Weiffenbach B, Keith T, Chu MP, Weaver A, et al. Dopamine D4 gene 7-repeat allele and attention deficit hyperactivity disorder. Am J Psychiatry. 1999 May;156(5):768-70.

Gilbert R, Widom CS, Browne K, Fergusson D, Webb E, Janson S. Burden and consequences of child maltreatment in high-income countries. Lancet. 2009 Jan;373(9657):68-81.

Gluckman PD, Hanson MA. The developmental origins of the metabolic syndrome. Trends Endocrinol Metab. 2004 May-Jun;15(4):183-7.

Hannon E, Lunnon K, Schalkwyk L, Mill J. Interindividual methylomic variation across blood, cortex, and cerebellum: implications for epigenetic studies of neurological and neuropsychiatric phenotypes. Epigenetics. 2015;10(11):1024-32.

Hedman E, Andersson E, Ljótsson B, Andersson G, Andersson E, Schalling M, et al. Clinical and genetic outcome determinants of Internet- and group-based cognitive behavior therapy for social anxiety disorder. Acta Psychiatr Scand. 2012 Aug;126(2):126-36.

Hofmann SG, Asnaani A, Vonk IJ, Sawyer AT, Fang A. The Efficacy of Cognitive Behavioral Therapy: A Review of Meta-analyses. Cognit Ther Res. 2012 Oct;36(5):427-40.

Hompes T, Izzi B, Gellens E, Morreels M, Fieuws $\mathrm{S}$, Pexsters A, et al. Investigating the influence of maternal cortisol and emotional state during pregnancy on the DNA methylation status of the glucocorticoid receptor gene (NR3C1) promoter region in cord blood. J Psychiatr Res. 2013 Jul;47(7):880-91.

Hudson JL, Lester KJ, Lewis CM, Tropeano M, Creswell C, Collier DA, et al. Predicting outcomes following cognitive behaviour therapy in child anxiety disorders: the influence of genetic, demographic and clinical information. J Child Psychol Psychiatry. 2013 Oct;54(10): 1086-94.

Jirtle RL, Skinner MK. Environmental epigenomics and disease susceptibility. Nat Rev Genet. 2007 Apr;8(4):253-62.

Joehanes R, Just AC, Marioni RE, Pilling LC, Reynolds LM, Mandaviya PR, et al. Epigenetic Signatures of Cigarette Smoking. Circ Cardiovasc Genet. 2016 Oct;9(5):436-47.

Karg K, Burmeister M, Shedden K, Sen S. The serotonin transporter promoter variant $(5-\mathrm{HT}$ TLPR), stress, and depression meta-analysis revisited: evidence of genetic moderation. Arch Gen Psychiatry. 2011 May;68(5):444-54.

Keers R, Coleman JR, Lester KJ, Roberts S, Breen G, Thastum M, et al. A Genome-Wide Test of the Differential Susceptibility Hypothesis Reveals a Genetic Predictor of Differential Response to Psychological Treatments for Child Anxiety Disorders. Psychother Psychosom. 2016;85(3):146-58.

Knuts I, Esquivel G, Kenis G, Overbeek T, Leibold N, Goossens L, et al. Therapygenetics: 5-HTTLPR genotype predicts the response to exposure therapy for agoraphobia. Eur Neuropsychopharmacol. 2014 Aug;24(8):1222-8.
Kohen R, Cain KC, Buzaitis A, Johnson V, Becker KJ, Teri L, et al. Response to psychosocial treatment in poststroke depression is associated with serotonin transporter polymorphisms. Stroke. 2011 Jul;42(7):2068-70.

Kumsta R, Marzi SJ, Viana J, Dempster EL, Crawford B, Rutter M, et al. Severe psychosocial deprivation in early childhood is associated with increased DNA methylation across a region spanning the transcription start site of CYP2E1. Transl Psychiatry. 2016 Jun;6(6):e830.

Kumsta R. The role of epigenetics for understanding mental health difficulties and its implications for psychotherapy research. Psychol Psychother. 2019 Jun;92(2):190-207.

Lau JY, Eley TC. The genetics of mood disorders. Annu Rev Clin Psychol. 2010;6(1):313-37.

Lesch KP, Bengel D, Heils A, Sabol SZ, Greenberg $\mathrm{BD}$, Petri S, et al. Association of anxiety-related traits with a polymorphism in the serotonin transporter gene regulatory region. Science. 1996 Nov;274(5292):1527-31.

Lester KJ, Eley TC. Therapygenetics: using genetic markers to predict response to psychological treatment for mood and anxiety disorders. Biol Mood Anxiety Disord. 2013 Feb;3(1):4.

Lester KJ, Roberts S, Keers R, Coleman JR, Breen $\mathrm{G}$, Wong CC, et al. Non-replication of the association between 5HTTLPR and response to psychological therapy for child anxiety disorders. Br J Psychiatry. 2016 Feb;208(2):182-8.

Loerinc AG, Meuret AE, Twohig MP, Rosenfield D, Bluett EJ, Craske MG. Response rates for CBT for anxiety disorders: need for standardized criteria. Clin Psychol Rev. 2015 Dec;42: 72-82.

Lonsdorf TB, Rück C, Bergström J, Andersson G, Ohman A, Lindefors N, et al. The COMTval158 met polymorphism is associated with symptom relief during exposure-based cognitive-behavioral treatment in panic disorder. BMC Psychiatry. 2010 Nov;10(1):99.

Lueken U, Zierhut KC, Hahn T, Straube B, Kircher T, Reif A, et al. Neurobiological markers predicting treatment response in anxiety disorders: A systematic review and implications for clinical application. Neurosci Biobehav Rev. 2016 Jul;66:143-62.

Marzi SJ, Sugden K, Arseneault L, Belsky DW, Burrage J, Corcoran DL, et al. Analysis of DNA Methylation in Young People: Limited Evidence for an Association between Victimization Stress and Epigenetic Variation in Blood. Am J Psychiatry. 2018 Jun;175(6):517-529.

Martin AR, Daly MJ, Robinson EB, Hyman SE, Neale, BM. Predicting polygenic risk of psychiatric disorders. Biol Psychiatry. 2019 Jul; 86(2):97-109.

Massart R, Dymov S, Millecamps M, Suderman M, Gregoire S, Koenigs K, et al. Overlapping signatures of chronic pain in the DNA methylation landscape of prefrontal cortex and peripheral T cells. Sci Rep. 2016 Jan;6(1):19615.

Monroe SM, Simons AD. Diathesis-stress theories in the context of life stress research: implications for the depressive disorders. Psychol Bull. 1991 Nov;110(3):406-25.

Murgatroyd C, Patchev AV, Wu Y, Micale V, Bockmühl Y, Fischer D, et al. Dynamic DNA methylation programs persistent adverse effects of early-life stress. Nat Neurosci. 2009 Dec;12(12):1559-66. 
Ouellet-Morin I, Wong CC, Danese A, Pariante CM, Papadopoulos AS, Mill J, et al. Increased serotonin transporter gene (SERT) DNA methylation is associated with bullying victimization and blunted cortisol response to stress in childhood: a longitudinal study of discordant monozygotic twins. Psychol Med. 2013 Sep;43(9):1813-23.

Perroud N, Salzmann A, Prada P, Nicastro R, Hoeppli ME, Furrer S, et al. Response to psychotherapy in borderline personality disorder and methylation status of the BDNF gene. Transl Psychiatry. 2013 Jan;3(1):e207.

Provençal N, Suderman MJ, Guillemin C, Massart $\mathrm{R}$, Ruggiero $\mathrm{A}$, Wang $\mathrm{D}$, et al. The signature of maternal rearing in the methylome in rhesus macaque prefrontal cortex and T cells. J Neurosci. 2012 Oct;32(44):15626-42.

Rayner C, Coleman JR, Purves KL, Hodsoll J, Goldsmith K, Alpers GW, et al. A genomewide association meta-analysis of prognostic outcomes following cognitive behavioural therapy in individuals with anxiety and depressive disorders. Transl Psychiatry. 2019 May;9(1):150.

Richlin VA, Arevalo JM, Zack JA, Cole SW. Stress-induced enhancement of NF-kB DNAbinding in the peripheral blood leukocyte pool: effects of lymphocyte redistribution. Brain Behav Immun. 2004 May;18(3):231-7.

Rijlaarsdam J, Pappa I, Walton E, BakermansKranenburg MJ, Mileva-Seitz VR, Rippe RC, et al. An epigenome-wide association metaanalysis of prenatal maternal stress in neonates: A model approach for replication. Epigenetics. 2016;11(2):140-9.

Risch N, Herrell R, Lehner T, Liang KY, Eaves L, Hoh J, et al. Interaction between the serotonin transporter gene (5-HTTLPR), stressful life events, and risk of depression: a meta-analysis. JAMA. 2009 Jun;301(23):2462-71.

Roberts S, Keers R, Breen G, Coleman JR, Jöhren P, Kepa A, et al. DNA methylation of FKBP5 and response to exposure-based psychological therapy. Am J Med Genet B Neuropsychiatr Genet. 2019 Mar;180(2):150-8.
Roberts S, Keers R, Lester KJ, Coleman JR, Breen G, Arendt K, et al. Hpa Axis Related Genes and Response to Psychological Therapies: genetics and Epigenetics. Depress Anxiety. 2015 Dec;32(12):861-70.

Roberts S, Lester KJ, Hudson JL, Rapee RM, Creswell C, Cooper PJ, et al. Serotonin transporter [corrected] methylation and response to cognitive behaviour therapy in children with anxiety disorders. Transl Psychiatry. 2014 Sep; 4(9):e444.

Rutter M. Maternal Deprivation Reassessed. Baltimore: Penguin Books; 1972.

Saunderson EA, Spiers H, Mifsud KR, GutierrezMecinas M, Trollope AF, Shaikh A, et al. Stress-induced gene expression and behavior are controlled by DNA methylation and methyl donor availability in the dentate gyrus. Proc Natl Acad Sci USA. 2016 Apr;113(17): $4830-5$.

Schlotz W, Phillips DI. Fetal origins of mental health: evidence and mechanisms. Brain Behav Immun. 2009 Oct;23(7):905-16.

Sonuga-Barke EJ, Kennedy M, Kumsta R, Knights N, Golm D, Rutter M, et al. Child-to-adult neurodevelopmental and mental health trajectories after early life deprivation: the young adult follow-up of the longitudinal English and Romanian Adoptees study. Lancet. 2017 Apr;389(10078):1539-48.

Stewart KR, Veselovska L, Kelsey G. Establishment and functions of DNA methylation in the germline. Epigenomics. 2016 Oct;8(10): 1399-413.

Strimbu K, Tavel JA. What are biomarkers?. Curr Opin HIV AIDS. 2010 Nov;5(6):463-466.

Taylor SE, Way BM, Welch WT, Hilmert CJ, Lehman BJ, Eisenberger NI. Early family environment, current adversity, the serotonin transporter promoter polymorphism, and depressive symptomatology. Biol Psychiatry. 2006 Oct;60(7):671-6.
Tobi EW, Slieker RC, Luijk R, Dekkers KF, Stein $\mathrm{AD}$, Xu KM, et al.; Biobank-based Integrative Omics Studies Consortium. DNA methylation as a mediator of the association between prenatal adversity and risk factors for metabolic disease in adulthood. Sci Adv. 2018 Jan; 4(1):eaao4364.

Trusheim MR, Berndt ER, Douglas FL. Stratified medicine: strategic and economic implications of combining drugs and clinical biomarkers. Nat Rev Drug Discov. 2007 Apr; 6(4):287-93.

Visscher PM, Wray NR, Zhang Q, Sklar P, McCarthy M, Brown MA, et al. 10 years of GWAS discovery: biology, function, and translation. Am J Hum Genet. 2017 Jul;101(1):5-22.

Wannemüller A, Moser D, Kumsta R, Jöhren HP, Margraf J. The Return of Fear: Variation of the Serotonin Transporter Gene Predicts Outcome of a Highly Standardized ExposureBased One-Session Fear Treatment. Psychother Psychosom. 2018;87(2):95-104.

Weaver IC, Cervoni N, Champagne FA, D'Alessio AC, Sharma S, Seckl JR, et al. Epigenetic programming by maternal behavior. Nat Neurosci. 2004 Aug;7(8):847-54.

Weisz JR, Kuppens S, Ng MY, Eckshtain D, Ugueto AM, Vaughn-Coaxum R, et al. What five decades of research tells us about the effects of youth psychological therapy: A multilevel meta-analysis and implications for science and practice. Am Psychol. 2017 Feb-Mar; 72(2):79-117.

Wong CC, Caspi A, Williams B, Craig IW, Houts $\mathrm{R}$, Ambler A, et al. A longitudinal study of epigenetic variation in twins. Epigenetics. 2010 Aug;5(6):516-26.

Yehuda R, Daskalakis NP, Desarnaud F, Makotkine I, Lehrner AL, Koch E, et al. Epigenetic Biomarkers as Predictors and Correlates of Symptom Improvement Following Psychotherapy in Combat Veterans with PTSD. Front Psychiatry. 2013 Sep;4:118.

Ziegler C, Richter J, Mahr M, Gajewska A, Schiele MA, Gehrmann A, et al. MAOA gene hypomethylation in panic disorder-reversibility of an epigenetic risk pattern by psychotherapy. Transl Psychiatry. 2016 Apr;6(4):e773. 